\title{
FORUM
}

\section{Polen nach den Wahlen - Außenpolitische Grundlinien der neuen Regierung}

\author{
Marek A. Cichocki*
}

Mit der Partei Recht und Gerechtigkeit (PiS) ist in Polen eine politische Kraft an die Macht gekommen, die sich nie intensiv mit Fragen der Außenpolitik befasst hat und die auch gar nicht geplant hatte, nach den Wahlen zum Sejm eine Rolle auf diesem Feld zu spielen. Führende Politiker der PiS konzentrierten sich vielmehr auf die Innenpolitik, besonders in den Bereichen Justiz und innere Sicherheit bzw. Bekämpfung der Korruption und Kriminalität. Ihre Aufgabe sieht sie vor allem in der Sanierung des ineffizienten, postkommunistischen Staates. Die Dinge haben sich allerdings wesentlich kompliziert nachdem die Koalitionsgespräche mit der liberalen Bürgerplattform (PO) misslangen und die PiS eine Minderheitsregierung bilden musste. Sie wird im Parlament von der klerikal-nationalistischen Liga der Polnischen Familien (CPR) und der populistischen, Selbstverteidigung ' (Samoobrona) gestützt. Die PiS steht jetzt vor der Herausforderung, Verantwortung für die polnische Außenpolitik zu übernehmen.

Trotz aller Vorbehalte gegenüber der PiS, die man in den letzten Monaten sehr oft besonders in der deutschen Presse finden konnte, deutet vieles darauf hin, dass keine grundsätzlichen Änderungen der polnischen Außenpolitik zu erwarten sind. Vielmehr ist anzunehmen, dass jene Elemente der polnischen Außenpolitik, die seit 2003 bestimmend geworden sind, mit bestimmten Modifikationen bestehen bleiben und weiterentwickelt werden. Hier sind besonders drei Tendenzen zu berücksichtigen:

- Polen wird immer intensiver für eine eigene aktive Rolle in der Ostpolitik der Union und ihrer Mitgliedstaaten werben und nach neuen Partnern suchen.

- In den innereuropäischen Angelegenheiten wird Polen weiter alle Möglichkeiten nutzen, seine eigene Position gegenüber anderen großen Mitgliedstaaten zu verbessern.

- Zwar werden die Kooperation mit den USA und die transatlantischen Beziehungen weiterhin als prioritär für die Sicherheit Polens verstanden, andererseits wächst der Druck, die bisherigen Beziehungen zu modifizieren.

\section{Osteuropa als Terrain der europapolitischen Profilbildung}

Seit dem Beitritt Polens zur Union hat der Wunsch, die polnische Ostpolitik in die gemeinsamen Mechanismen und Institutionen der Europäischen Union einzubinden, in Warschau geradezu den Rang einer außenpolitischen Doktrin erlangt. Das Ziel, die polnische Politik in Osteuropa zu einer Aufgabe der Union zu machen, entspringt der Einschätzung, dass Polen die Möglichkeit eigenständig oder auch in einem regionalen Kontext in Osteuropa zu agieren vor dem EU-Beitritt im Wesentlichen erschöpft hat. Zudem war Polen in dieser Hinsicht nur begrenzt erfolgreich, wie das Beispiel der Beziehungen zu Kutschmas Ukraine zeigte. Zweitens wird die Rolle, die Polen beim friedlichen Ausgang der so genannten ,Orangen Revolution“ in der Ukraine 2004/2005 spielte, in Warschau als positives Zu-

Dr. Marek A. Cichocki, Europäisches Zentrum Natolin, Warschau. 
kunftsmodell für eine Kooperation zwischen EU-Institutionen (Javier Solana) und interessierten EU-Mitgliedern (hier vor allem die baltischen Staaten, Polen, die Slowakei und Deutschland) angesehen, die bei der Unterstützung demokratischer Veränderungen und beim Krisenmanagement in Osteuropa zusammen wirken. Drittens hat die Art und Weise, wie das Projekt der Ostseepipeline zwischen Berlin und Moskau verhandelt worden ist, die Schwäche der polnischen Position zum Vorschein gebracht und die Aufmerksamkeit darauf gelenkt, dass Polen neue Verbündete für die Ostpolitik (gegenüber der Ukraine und Russland) suchen muss.

Vielfach erklärte die neue PiS-Regierung, wie notwendig die Einbindung der Politik Polens gegenüber Osteuropa in den breiteren politischen Kontext der Union ist. Außenminister Meller betonte im Außenpolitischen Ausschuss des Sejms, Ministerpräsident Marcinkiewicz beim ersten Treffen mit dem diplomatischen Korps und Staatspräsident Kaczynski noch während des Wahlkampfes, dass die enge Kooperation zwischen Polen, den anderen EUStaaten und den EU-Institutionen in Fragen der europäischen Ostpolitik zu den wichtigsten Prioritäten Polens in der Union zählt. Von Marcinkewicz wurde das mit der Forderung nach einer gemeinsamen Energiepolitik und der Vertiefung der GASP verbunden.

Das Drängen der polnischen Seite, zu einer vertieften Kooperation in der Ostpolitik zu gelangen, ist in erster Linie als eine Bewährungsprobe für die Solidarität zwischen alten und neuen EU-Mitgliedern zu verstehen. Die russischen Versuche, die politische Kohärenz der erweiterten Union auf die Probe zu stellen und damit die Stärke der Solidarität zwischen den alten und neuen Mitgliedern zu testen, werden in Warschau mit größter Sorge beobachtet. In diesem Kontext sollten auch die Kritik am deutsch-russischen Pipelineprojekt sowie die Vorschläge in Richtung einer stärkeren Koordinierung der Energiepolitik der Mitgliedstaaten, die polnische Abgeordnete im Europäischen Parlament angesichts des russisch-ukrainischen Energiekonflikts präsentierten, gedeutet werden. Darüber hinaus legt Warschau großen Wert darauf, in seiner Außenpolitik (hier in ihrer östlichen Dimension) nicht von anderen Mitgliedstaaten in seinem Handlungsspielraum eingeschränkt zu werden. So wird die Maxime verfolgt, dass eine vertiefte Kooperation in der Ostpolitik nicht unbedingt eine Zusage polnischer Politiker zu neuen Verfahren und anderen Reformelementen des Verfassungsvertrags bedeutet, beispielsweise die Position eines europäischen Außenministers einzuführen. Man ist sich in Warschau bewusst, dass der polnische Ehrgeiz für eine aktive und von Polen beeinflusste europäische Ostpolitik bei den alten EU-Mitgliedern nicht auf große Sympathie stößt. Ähnlich wie die Union auf die ,Orange Revolution' in der Ukraine reagierte - Desinteresse von Frankreich und eine reaktive Politik Deutschlands - wird sie wahrscheinlich auch künftig in Sachen Ostpolitik agieren, so jedenfalls die Lehre, die die neue polnische Regierung zieht. Polen wird alle Mechanismen vermeiden wollen, die potenziell seinen Spielraum in Osteuropa reduzieren würde.

\section{Die schwierige Suche nach verlässlichen Koalitionen}

Für einen Politiker, der in der ausländischen Presse oft einer geradezu gefährlichen Distanz zur Europäischen Union verdächtigt wurde, reist Marcinkiewicz überraschend oft nach Brüssel. Das tat er noch als designierter Ministerpräsident vor den Präsidentschaftswahlen im Oktober 2005. Brüssel war erneut das Ziel seines ersten offiziellen Besuches, nachdem er und seine Regierung im Sejm bestätigt worden waren.

Der Grund dafür ist vor allem der politische Druck, der aus der mangelnden Kompromissbereitschaft der Regierungen der EU-Staaten bezüglich der Finanziellen Vorausschau für 2007-2013 resultierte, weniger war es die Absicht sein nicht gerade positives europäisches Image in Brüssel aufzubessern. Der stärkste Druck kam überraschenderweise aus Lon- 
don. Die britische Regierung hatte in ihrem Kompromissentwurf vom 5. Dezember 2005 Kürzungen im EU-Haushalt vorgeschlagen, die Polen ungefähr 6 Milliarden Euro gekostet hätten. Angesichts dieser Gefahr griff die polnische Regierung zu der Methode, die man seit dem EU-Beitritt mit mäßigem Erfolg zu praktizieren versuchte, nämlich eine Koalition der Unzufriedenen zu bilden. Dabei verhandelte man besonders intensiv mit den Visegradstaaten. Um auf der Bühne, die von den großen Mitgliedstaaten beim Drama um den Haushalt besetzt wurde, eine eigene Rolle zu spielen, signalisierte Marcinkiewicz, dass Polen bereit wäre, für eine bessere Finanzielle Vorausschau für die Jahre 2007-2013 Vorschläge für eine tiefergehende Reform der Gemeinsamen Agrarpolitik zu unterstützen. Dies alles kam aber offenbar zu spät, da sich die Briten schon im November darauf konzentriert hatten, anderen Mitgliedstaaten den Schwarzen Peter für ihre miserable Präsidentschaft und mangelnde Führungsfähigkeit zuzuschieben und das Problem der grundsätzlichen Haushaltsreform aufzuschieben.

Die Situation, in der sich die neue polnische Regierung in der Debatte um den EU-Haushalt Ende 2005 gefunden hat, zeigt das Dilemma der polnischen Europapolitik nach dem EU-Beitritt. Sie illustriert nämlich die Bemühungen, die Europapolitik Polens auf den Ansatz variabler Koalitionen innerhalb der Union zu stützen. Dieser Gedanke war besonders stark von den Politikern der Bürgerplattform, Jan Maria Rokita und Donald Tusk, im Kontext der Verfassungsdebatte propagiert worden. Er liegt ebenso den Politikern der PiS nahe und wurde vor wenigen Wochen von Außenminister Meller im Sejm bestätigt. Konkret bedeutet der Ansatz der variablen Koalitionen: In der Europapolitik hat Polen keine festen Verbündeten, sondern nur eigene Interessen, für die Mehrheiten gesucht werden müssen. Dies führt dazu, dass sich Polen im Konfliktfall in der Rolle sieht, der Stifter einer Koalition der kleineren und mittelgroßen unter den neuen Mitgliedern zu sein. Im Grunde genommen ist das ein Ansatz, den Polen schon in der Endphase der Beitrittverhandlungen vor dem Kopenhagener Gipfel im Dezember 2002 praktiziert hatte. Ähnliches wurde in der Auseinandersetzung um den Verfassungsentwurf des Konvents versucht. Zu diesem Mittel griff die neue polnische Regierung jetzt sowohl in der Frage des Haushaltes wie in der Auseinandersetzung um die deutsch-russische Pipeline. Aber die Schwäche dieses Ansatzes scheint offenkundig: Die so geschlossenen Koalitionen erweisen sich als sehr brüchig, und in der Endphase von Auseinandersetzungen werden die kleineren Mitgliedstaaten gegeneinander ausgespielt während die Großen die Entscheidung treffen.

Nur einmal ist es Polen beinahe gelungen, die alten Spielregeln in der Union durcheinander zu bringen, als es zu einer Allianz zwischen Polen und Spanien gegen die doppelte Mehrheit gekommen ist. Wegen der Ereignisse im März 2004, die zu einem Regierungswechsel in Spanien beitrugen, ist diese Kooperation leider zu Ende gegangen. Dennoch, um ihre Position wesentlich zu verbessern muss die polnische Politik nicht nur die Fähigkeit besitzen, ad hoc Koalitionen unter den kleineren Newcomern aus Mittel- und Osteuropa zu bilden, sondern auch mit den großen Mitgliedsländern einen modus operandi zu erarbeiten. Daher hat die neue polnische Regierung mit gemischten Gefühlen die Überlegungen ${ }^{1}$ von Philippe Douste-Blazy und Christoph Heusgen aufgegriffen, die trilaterale Zusammenarbeit mit Polen in Form der alten Idee des Weimarer Dreiecks zu beleben. Die polnische Politik sucht dringend neue Wege, effizienter gegenüber den großen EU-Mitgliedern zu sein. Aber offensichtlich sieht sie die Lösung in einer breiteren Form, als sie das Weimarer Dreieck bieten könnte. Darin liegt wahrscheinlich der Grund, warum die neue Regierung der Zusam-

1 Über die Einzelinitiativen siehe: Philippe Douste-Blazy, In gemeinsamer Verantwortung, FAZ, 24. Nov. 2005; Petra Pinzler, Merkels Welt-Erklärer, Die Zeit, 47/2005. 
menarbeit im Rahmen der G6 (Frankreich, Deutschland, Spanien, Polen, Großbritannien und Italien) mehr Bedeutung beimessen dürfte, die zunächst vor allem Fragen der Innenund Justizpolitik gewidmet sein sollte, doch mit der Zeit und nach Bedarf um andere Politiken in der Union angereichert oder ausgebaut werden könnte.

Der Ausgang der Verhandlungen über den EU-Haushalt 2007-2013 im Rahmen der britischen Präsidentschaft wird wichtige Auswirkungen auf die Europapolitik der neuen Regierung haben. Die Vorschläge Londons, die anachronistische Haushaltstruktur mit Kürzungen bei den Strukturfonds zu retten, die vor allem die neuen Mitgliedsländer (gerade in den ersten Jahren ihrer Mitgliedschaft) stark getroffen hätten, waren für Warschau wie eine kalte Dusche. Es hat sich herausgestellt, dass Polen ohne das Zusammenwirken mit Frankreich und Deutschland nicht im Stande gewesen wäre, seine Interessen in den Haushaltsverhandlungen durchzusetzen. Zumindest ein Teil der Rechten in der polnischen Politik vertrat immer die Ansicht, England sei der beste Verbündete Polens in Europa, wegen der politischen Affinität in Sachen transatlantischer Beziehungen und wegen eines ähnlichen Grundverständnisses bei der Beurteilung politischer Prozesse in Europa und in der Welt. Die Rolle, die die britische Regierung in den Verhandlungen über den Verfassungsvertrag gespielt hat, wurde in Polen schlichtweg übersehen. Angesichts der britischen Taktik in den Verhandlungen über den EU-Haushalt wird es aber künftig sehr schwierig, diese alte Auffassung unangefochten zu vertreten. Die neue polnische Regierung wird gezwungen sein, die Position Polens in der Europapolitik und im Hinblick auf Koalitionspartner neu zu definieren.

\section{Sicherheitspolitik: Modifizierung der proatlantischen Haltung?}

$\mathrm{Zu}$ Recht ist angenommen worden, dass die neue polnische Regierung eine eindeutig proatlantistische Stellung beziehen wird. In dieser Hinsicht wird sie eine Fortsetzung der Politik der Vorgänger (Leszek Miller, Marek Belka, Aleksander Kwasniewski) praktizieren, allerdings mit bestimmten Modifikationen. In seiner ersten Regierungserklärung hat Marcinkiewicz eindeutig festgestellt, die Kooperation mit den USA sei für Polen der Grundstein der nationalen Sicherheit. Die Nominierung von Radoslaw Sikorski als neuer Verteidigungsminister, der früher in Washington im Milieu der Neocons im American Enterprise Institute tätig war, bestätigte nur den Eindruck, Polen werde seine Abhängigkeit von den USA in Fragen der Sicherheitspolitik noch weiter vertiefen wollen.

Hier ist aber eher eine gegenläufige Tendenz zu erwarten. Zunächst hat sich die neue Regierung ganz zu Beginn öffentlich für die Beteiligung Polens am Raketenabwehrprogramm MDI ausgesprochen. Die Erklärungen zu dem Projekt wurden aber sehr schnell abgeschwächt, nachdem die polnische Öffentlichkeit die Idee der polnischen Beteiligung am MDI-Programm eher kritisch aufgenommen hat. Jedenfalls wurden Fragen aufgeworfen, ob Polen in der Kooperation mit den USA wirklich so weit gehen sollte. Die unter der SLD-Regierung (postkommunistische Linke) nur gedämpft geführte Diskussion über die Zukunft der polnischen Unterstützung für die USA drängt jetzt viel stärker ans Tageslicht und übt zusätzlichen Druck auf die neue Regierung aus. Ferner muss festgestellt werden, dass gerade unter den polnischen Rechten trotz der deklaratorischen Unterstützung für die USA die Auffassung immer mehr Anhänger findet, die Beziehungen zwischen Warschau und Washington müssten pragmatischer und eigenständiger gestaltet werden. Verstärkt wird diese kritische Einschätzung durch die Art und Weise, in der Polen in Folge der innenpolitischen Auseinandersetzungen in Washington um die angeblichen geheimen Gefängnisse der CIA in Ostmitteleuropa in das Feuer der internationalen Kritik geraten ist.

Polen wird seine Verpflichtungen (weiteres militärisches Engagement im Irak, Beteiligung am ISAF-Einsatz in Afghanistan ab 2007) sicherlich nicht in Frage stellen, aber der 
Druck, die Bedingungen der Kooperation nach neuen, mehr partnerschaftlichen Grundsätzen zu gestalten, wird deutlich zunehmen. Schon während eines ersten Besuches in den USA legte Verteidigungsminister Radoslaw Sikorski im Dezember 2005 der amerikanischen Administration eine Liste mit Vorschlägen vor, die die Kooperation bei der Modernisierung der polnischen Streitkräfte verbessern sollten. Man erwartet insofern viel mehr Engagement seitens der Amerikaner, darunter die Schaffung einer polnisch-ukrainisch-amerikanischen Brigade, die der transatlantischen Kooperation in Osteuropa eine konkrete Form geben würde.

\section{Ausblick}

Die ersten Schritte der neuen Regierung zeigen, dass die polnische Außenpolitik viel konstanter geworden ist als es oftmals in kritischen Analysen geschildert wird. Die neuen innenpolitischen Faktoren verändern die Grundausrichtung der polnischen Außen- und Europapolitik nicht. In der Periode vor dem Beitritt war die Integration in die NATO und die Europäische Union der Hauptwegweiser der polnischen Außenpolitik. Inzwischen, nach dem Beitritt, bildet sich allmählich eine neue Interessendefinition heraus, die trotz aller politischen Unterschiede unter den Parteien bestimmend geworden ist. Sie betrifft eine aktive Rolle für die Entwicklung der östlichen Dimension der Union, die Etablierung einer starken polnischen Position innerhalb der Europäischen Union durch variable Koalitionen sowie eine Modifizierung der Kooperation mit den USA.

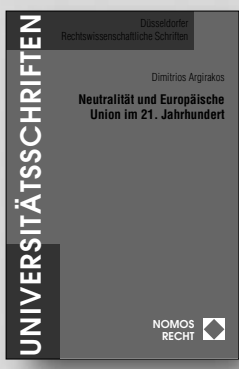

\section{Neutralität und Europäische Union im 21. Jahrhundert}

Von RA Dr. Dimitrios Argirakos

2005, 331 S., brosch., 59,- $€$, ISBN 3-8329-1396-3

(Düsseldorfer Rechtswissenschaftliche Schriften, Bd. 33)

Jede Epoche in der Geschichte hatte ihre Art der Neutralität. Dieses Buch bietet angesichts dramatischer und weitreichender Veränderungen im internationalen politischen Geschehen nicht nur eine sachliche und umfassende Orientierung über die Möglichkeit der Vereinbarkeit eines Neutralitätsstatus mit einer EU-Mitgliedschaft, sondern zeigt darüber hinaus auch einen Weg für den Fortbestand eines völkerrechtlichen Neutralitätsgedankens in einer Zeit, in der es sich für oder gegen den internationalen Terrorismus zu entscheiden gilt. 